University of Texas at El Paso

ScholarWorks@UTEP

6-2007

\title{
From (Idealized) Exact Causality-Preserving Transformations to Practically Useful Approximately-Preserving Ones: A General Approach
}

\author{
Vladik Kreinovich \\ The University of Texas at El Paso, vladik@utep.edu \\ Olga Kosheleva \\ The University of Texas at El Paso, olgak@utep.edu
}

Follow this and additional works at: https://scholarworks.utep.edu/cs_techrep

Part of the Computer Engineering Commons

Comments:

Technical Report: UTEP-CS-07-35

Published in International Journal of Theoretical Physics, 2008, Vol. 27, No. 4, pp. 1083-1091.

\section{Recommended Citation}

Kreinovich, Vladik and Kosheleva, Olga, "From (Idealized) Exact Causality-Preserving Transformations to Practically Useful Approximately-Preserving Ones: A General Approach" (2007). Departmental Technical Reports (CS). 160.

https://scholarworks.utep.edu/cs_techrep/160

This Article is brought to you for free and open access by the Computer Science at ScholarWorks@UTEP. It has been accepted for inclusion in Departmental Technical Reports (CS) by an authorized administrator of ScholarWorks@UTEP. For more information, please contact Iweber@utep.edu. 


\title{
From (Idealized) Exact Causality-Preserving Transformations to Practically Useful Approximately-Preserving Ones: A General Approach
}

\author{
Vladik Kreinovich and Olga Kosheleva \\ University of Texas at El Paso, El Paso, TX 79968, USA \\ vladik@utep.edu, olgak@utep.edu
}

\begin{abstract}
It is known that every causality-preserving transformation of Minkowski space-time is a composition of Lorentz transformations, shifts, rotations, and dilations. In principle, this result means that by only knowing the causality relation, we can determine the coordinate and metric structure on the space-time. However, strictly speaking, the theorem only says that this reconstruction is possible if we know the exact causality relation. In practice, measurements are never $100 \%$ accurate. It is therefore desirable to prove that if a transformation approximately preserves causality, then it is approximately equal to an above-described composition.

Such a result was indeed proven, but only for a very particular case of approximate preservation.

In this paper, we prove that simple compactness-related ideas can lead to a transformation of the exact causality-preserving result into an approximately-preserving one.
\end{abstract}

Causality-preserving mappings: formulation of the general problem. One of the fundamental notions of physics is the notion of causality, the description of which events can causally influence others. In particular, the Minkowski space-time of special relativity is an $(n+1)$-dimensional space-time $E=R^{n+1}$, in which the causality relation $a \leq b$ between events $a=\left(a_{0}, a_{1}, \ldots, a_{n}\right) \in E$ and $b=\left(b_{0}, b_{1}, \ldots, b_{n}\right) \in E$ is described by the formula

$$
a \leq b \leftrightarrow a=b \vee\left(b_{0} \geq a_{0} \&(b-a)^{2} \geq 0\right),
$$

where $a^{2} \stackrel{\text { def }}{=} a_{0}^{2}-a_{1}^{2}-\ldots-a_{n}^{2}$.

It is known that for every $n \geq 2$, every bijection $E \rightarrow E$ which preserves the Minkowski causality relation is linear (moreover, it is a composition of Lorentz transformations, shirts, rotations, and dilations). This theorem was first proven by A. D. Alexandrov [1, 5]; see also [2, 3, 7, 8, 16, 22, 26, 27, 29, 30, 31, 32, 33, 35]. 
The original Alexandrov's theorem requires that the causality is preserved for all pairs of events $a \leq b$. In practice, however, all our measurements are restricted only to a bounded domain. For causality-preserving transformations on a bounded domain, a similar result was only proven by A. D. Alexandrov; see, e.g., $[4,16]$. For bounded domains, in addition to linear transformations, we also have special nonlinear transformations - inversions:

\section{Definition 1.}

- A Lorentz transformation is a mapping

$$
\left(a_{0}, \vec{a}\right) \rightarrow\left(\frac{a_{0}-\vec{v} \cdot \vec{a}}{1-\vec{v} \cdot \vec{v}}, \frac{\vec{a}-a_{0} \cdot \vec{v}}{1-\vec{v} \cdot \vec{v}}\right)
$$

where $\vec{v} \cdot \vec{a} \stackrel{\text { def }}{=} v_{1} \cdot a_{1}+\ldots+v_{n} \cdot a_{n}$, and $\vec{v} \cdot \vec{v} \leq 1$.

- $A$ rotation is a mapping $\left(a_{0}, \vec{a}\right) \rightarrow\left(a_{0}, T a\right)$, where $T$ is a rotation in the $n$-dimensional Euclidean space.

- A shift is a mapping $a \rightarrow a+b$, for some $b \in E$.

- A dilation is a mapping $a \rightarrow \lambda \cdot a$, for some real number $\lambda$.

- An inversion is a mapping $a \rightarrow \frac{a-b}{(a-b)^{2}}+b$, for some $b \in E$.

- A singular double inversion is a mapping

$$
a \rightarrow \frac{(a-b)+c \cdot(a-b)^{2}}{1+2 \cdot c(a-b)}+b
$$

for some $b \in E$ and $c \in E$ for which $c^{2}=0$.

- By a conformal mapping, we mean one of the above transformations or their composition.

Comment. In the following text, we will use the fact that conformal mappings are described by a finite number of parameters.

\section{Definition 2.}

- By a bounded domain D, we mean a closure of a bounded open set.

- Let $D$ be a bounded domain. We say that a bijection $f: D \rightarrow D$ preserves causality if it has the following two properties:

- for every two events $a, b \in D, a \leq b$ implies $f(a) \leq f(b)$, and

- for every two events $a, b \in D, a \leq b$ implies $f^{-1}(a) \leq f^{-1}(b)$. 
Theorem. $[4,16]$ Let $D$ be a bounded domain. Then, every bijection $f: D \rightarrow$ $D$ which preserves causality is a conformal mapping.

Discussion: it is desirable to make this result more practical. In principle, this result means by knowing only the causality relation, we can determine the detailed structure on the space-time; see, e.g., [22]. However, strictly speaking, the theorem only says that this reconstruction is possible if we know the exact causality relation. In practice, measurements are never absolutely exact. It is therefore desirable to prove that if a transformation approximately preserves causality, then it is approximately conformal.

Crudely speaking, we would like to show that if we want to reconstruct the structure with accuracy $\varepsilon>0$, then we can find such a measurement accuracy $\delta$ that if we can determine causality with accuracy $\delta$, then based on this approximate causality, we will be able to reconstruct the structure with the desired accuracy $\varepsilon$. (In the following text, we will give precise definitions; right now, we just want to informally explain what we want.)

What was known. One approximate-preservation result was indeed obtained in [24], but for a very particular case of approximate causality preservation. It is therefore desirable to get a more general results.

Our main idea. The result from [24] required, in effect, a radically new proof of the causality-preservation result, and the ideas from this proof are not easy to generalize further; see, e.g., [20].

Instead of looking for drastically new proof ideas, we will apply a general approach (see, e.g., $[18,19]$ ), according to which we analyzing the existing results (and sometimes the existing proofs of these results) and use general techniques to extract stronger versions of these results. In particular, in this paper, we use simple compactness-related techniques to design the desired $\varepsilon-\delta$-version of the Alexandrov's theorem.

Physical motivations for the following definitions. We want to reconstruct the events and the causality relation with a certain accuracy.

Let us start with reconstructing events, i.e., points from the set $E$. For every real number $\varepsilon>0$, it is natural to say that the events $a=\left(a_{0}, a_{1}, \ldots, a_{n}\right)$ and $b=\left(b_{0}, b_{1}, \ldots, b_{n}\right)$ are $\varepsilon$-close if all their coordinates are $\varepsilon$-close, i.e., if $\left|a_{i}-b_{i}\right| \leq$ $\varepsilon$ for all $i$. We will denote this closeness by $a \approx_{\varepsilon} b$. This condition is equivalent to $\max _{i}\left|a_{i}-b_{i}\right| \leq \varepsilon$. Thus, it is reasonable to take $\|a-b\| \stackrel{\text { def }}{=} \max _{i}\left|a_{i}-b_{i}\right|$ as the natural measure of distance between the two events.

Now, we can talk about approximate causality. It is reasonable to say that an event $a \varepsilon$-approximately causally precedes $b$ if there exists events $a^{\prime}$ and $b^{\prime}$ such that $a^{\prime}$ is $\varepsilon$-close to $a, b^{\prime}$ is $\varepsilon$-close to $b$, and $a^{\prime}$ causally precedes $b^{\prime}\left(a^{\prime} \leq b^{\prime}\right)$. We will denote this approximate causality by $a \leq_{\varepsilon} b$.

Finally, let us make some comments about the mapping $f$. In physics, as we have mentioned, all measurements are approximate; thus, for any function $f(x)$ 
with a physical meaning to have practical sense, we must make sure that we can determine the value of this function based on the approximate value $x^{\prime} \approx x$ of the input. To be more precise, for this function to be practically useful, we must know, for every desired accuracy $\varepsilon$, with what accuracy $\delta$ we must measure $x$ to be able to determine $f(x)$ with the desired accuracy. In other words, we must know the dependence of $\delta$ on $\varepsilon$, i.e., the bound on the modulus of continuity of the desired function.

Thus, we arrive at the following definitions.

Definition 3. For every point $a=\left(a_{0}, \ldots, a_{n}\right) \in E$, we define $\|a\|=\max _{i}\left|a_{i}\right|$.

Definition 4. Let $\delta>0$ be a real number.

- We say that the points $a, a^{\prime} \in E$ are $\delta$-close if $\left\|a-a^{\prime}\right\| \leq \delta$. We will denote this relation by $a \approx_{\delta} a^{\prime}$.

- We say that an event $a \in E \delta$-approximately precedes an event $b \in E$ if there exist events $a^{\prime}, b^{\prime} \in E$ such that $a^{\prime}$ is $\delta$-close to $a, b^{\prime}$ is $\varepsilon$-close to $b$, and $a^{\prime} \leq b^{\prime}$. We will denote this relation by $a \leq_{\delta} b$.

\section{Definition 5.}

- By a modulus of continuity, we mean a mapping $m(\varepsilon)$ which maps positive real numbers into positive real numbers.

- Let $m$ be a given modulus of continuity. We say that a bijection $f: D \rightarrow D$ is $m$-continuous if for every $\varepsilon>0$ and for all $a, a^{\prime} \in D$, the following two properties hold:

- if $\left\|a-a^{\prime}\right\| \leq m(\varepsilon)$, then $\left\|f(a)-f\left(a^{\prime}\right)\right\| \leq \varepsilon ;$ and

- if $\left\|a-a^{\prime}\right\| \leq m(\varepsilon)$, then $\left\|f^{-1}(a)-f^{-1}\left(a^{\prime}\right)\right\| \leq \varepsilon$.

Definition 6. Let $D$ be a bounded domain. and let $\delta>0$ be a real number. We say that a bijection $f: D \rightarrow D \delta$-preserves causality if it has the following two properties:

- for every two events $a, b \in D, a \leq b$ implies $f(a) \leq_{\delta} f(b)$, and

- for every two events $a, b \in D, a \leq b$ implies $f^{-1}(a) \leq_{\delta} f^{-1}(b)$.

Definition 7. Let $\varepsilon>0$ be a real number. We say that a function $f: D \rightarrow D$ is $\varepsilon$-conformal if there exists a conformal mapping $c: D \rightarrow D$ such that for every $a \in D$, we have $f(a) \approx_{\varepsilon} c(a)$.

Proposition 1. Let $D$ be a bounded domain, and let $m$ be a modulus of continuity. Then, for every $\varepsilon>0$, there exists a $\delta>0$ such that if an $m$-continuous bijection $f: D \rightarrow D \delta$-preserves causality, then $f$ is $\varepsilon$-conformal. 


\section{Proof.}

$1^{\circ}$. Let us start with some notations and observations. By definition, a mapping $f$ is $\varepsilon$-conformal if there exists a conformal mapping $c$ for which $\|f(a)-c(a)\| \leq \varepsilon$ for all events $a \in D$. This condition is equivalent to $\|f-c\|_{\infty} \leq \varepsilon$, where for every function $g(a)$, we denote $\|g\|_{\infty} \stackrel{\text { def }}{=} \max _{a \in D} g(a)$.

The condition that $\|f-c\|_{\infty} \leq \varepsilon$ for some conformal transformation $c$ is, in its turn, equivalent to $d(f, \mathcal{C}) \leq \varepsilon$, where $d(f, \mathcal{C}) \stackrel{\text { def }}{=} \min _{c \in \mathcal{C}}\|f-c\|_{\infty}$ is the distance from $f$ to the set $\mathcal{C}$ of all conformal mappings. Since the set $\mathcal{C}$ is finitedimensional, this distance $d(\cdot, \mathcal{C})$ is continuous in terms of the sup metric $\|\cdot\|_{\infty}$.

$2^{\circ}$. Let us now prove our result by reduction to a contradiction. Let us assume that for some $\varepsilon>0$, no such $\delta>0$ exists. This means that for every natural number $k$, for $\delta=1 / k$, there exists an $m$-continuous bijection $f_{k}: D \rightarrow D$ which $(1 / k)$-preserves causality but which is not $\varepsilon$-conformal, i.e., $d\left(f_{k}, \mathcal{C}\right) \geq \varepsilon$.

The set of all $m$-continuous functions $f: D \rightarrow D$ is uniformly continuous (by definition of $m$-continuity) and equibounded (since $f(a) \in D$ for all $a$, and $D$ is a bounded domain). Thus, in terms of the sup metric, it is a compact set. So, from the sequence $f_{k}$, we can extract a convergent subsequence.

Since the inverse mappings also belong to the same compact set, from this subsequence, we can extract a sub-subsequence for which the inverse functions converge as well.

To simplify notations (and without losing generality), we can denote this convergent sub-subsequence by the same notations $f_{k}$. Then,

- $f_{k} \rightarrow f$ for some function $f$,

- $f_{k}^{-1} \rightarrow f^{\prime}$ for some function $f^{\prime}$,

- each mapping $f_{k} \frac{1}{k}$-preserves causality, and

- each mapping $f_{k}$ is not $\varepsilon$-conformal, i.e., $d\left(f_{k}, \mathcal{C}\right) \geq \varepsilon$.

$3^{\circ}$. For every point $a$, we have $f_{k}^{-1}\left(f_{k}(a)\right)=a$. Thus, in the limit $k \rightarrow \infty$, we get $f^{\prime}(f(a))=a$ and similarly, $f\left(f^{\prime}(a)\right)=a$. So, $f^{\prime}$ is the inverse function to $f$ : $f^{\prime}=f^{-1}$.

$4^{\circ}$. Since $d\left(f_{k}, \mathcal{C}\right) \geq \varepsilon$ and $f_{k} \rightarrow f$, in the limit, we get $d(f, \mathcal{C}) \geq \varepsilon$.

$5^{\circ}$. Let us show that the limit transformation $f$ preserves causality, i.e., that for all $a$ and $b$ for which $a \leq b$, we have $f(a) \leq f(b)$ and $f^{-1}(a) \leq f^{-1}(b)$.

$5 \cdot 1^{\circ}$. Let us first prove the first implication. Let us fix $a$ and $b$ for which $a \leq b$.

Since the function $f_{k} \frac{1}{k}$-preserves causality, for every $a$ and $b$, we have $f_{k}(a) \leq_{1 / k} f_{k}(b)$. By definition of approximate causality, this means that there exist the events $A_{k} \leq B_{k}$ for which $\left\|f_{k}(a)-A_{k}\right\| \leq 1 / k$ and $\left\|f_{k}(b)-B_{k}\right\| \leq 1 / k$. 
When $k \rightarrow \infty$, then $f_{k}(a) \rightarrow f(a)$; since $\left\|f_{k}(a)-A_{k}\right\| \leq 1 / k$, we also have $A_{k} \rightarrow f(a)$. Similarly, we have $B_{k} \rightarrow f(b)$. The Minkowski causality relation is closed, so $A_{k} \leq B_{k}$ implies that $\lim A_{k} \leq \lim B_{k}$, i.e., that $f(a) \leq f(b)$.

$5.2^{\circ}$. Similarly, we prove that $a \leq b$ implies $f^{-1}(a) \leq f^{-1}(b)$.

Thus, $f$ preserves causality and hence, according to Alexandrov's theorem, $f$ is a conformal mapping, i.e., $f \in \mathcal{C}$. This contradicts to our conclusion that $d(f, \mathcal{C}) \geq \varepsilon>0$. The proposition is proven.

Comment. It is worth mentioning that most applications described in $[18,19]$ use much more sophisticated techniques than our simple proof. It is also worth mentioning that some more more sophisticated logical techniques have been used in geometry [25].

Applicability to curved space-time models. The Minkowski space-time of special relativity is a good approximation to the actual space-time. However, the actual space-time is different from the Minkowski space-time: e.g., it is curved. A natural question is: can we extend the above result to curves spaces?

Some results of this type are known; see, e.g., $[11,12,13,14,15,16]$; however, these results only handle specific type of curved spaces, and besides - just like the original Alexandrov's theorem - they have only been proven for the case when the transformations exactly preserve causality. We will show that is possible to extend our results for all space-time models which are sufficiently close to the Minkowski space-time. Let us first extend the above definitions to a general causality relation.

Definition 8. Let $D$ be a bounded domain. By a causality relation, we mean an order $\preceq$ on $D$.

Definition 9. Let $D$ be a bounded domain, let $\delta>0$ be a real number, and let $\preceq$ be a causality relation on $D$.

- We say that an event $a \in E \delta$-approximately precedes an event $b \in E$ if there exist events $a^{\prime}, b^{\prime} \in E$ such that $a^{\prime}$ is $\varepsilon$-close to $a, b^{\prime}$ is $\varepsilon$-close to $b$, and $a^{\prime} \preceq b^{\prime}$. We will denote this relation by $a{ }_{\delta} b$.

- We say that a bijection $f: D \rightarrow D \delta$-preserves causality $\preceq$ if it has the following two properties:

- for every two events $a, b \in D, a \preceq b$ implies $f(a) \preceq_{\delta} f(b)$, and

- for every two events $a, b \in D, a \preceq b$ implies $f^{-1}(a) \preceq_{\delta} f^{-1}(b)$.

- We say that a causality relation $\preceq$ on a bounded domain $D$ is $\delta$-close to the Minkowski order $\leq$ if for all $a, b \in D$, the following two properties hold:

- if $a \leq b$, then $a \preceq_{\delta} b$;

- if $a \preceq b$, then $a \leq{ }_{\delta} b$. 
Proposition 2. Let $D$ be a bounded domain, and let $m$ be a modulus of continuity. Then, for every $\varepsilon>0$, there exists $a \delta>0$ such that if a causality relation $\preceq$ is $\delta$-close to the Minkowski relation $\leq$, and an $m$-continuous bijection $f: D \rightarrow D \delta$-preserves the relation $\preceq$, then $f$ is $\varepsilon$-conformal.

\section{Proof.}

$1^{\circ}$. Let us prove our result by reduction to a contradiction. Let us assume that for some $\varepsilon>0$, no such $\delta>0$ exists. This means that for every natural number $k$, for $\delta=1 / k$, there exists an $m$-continuous bijection $f_{k}: D \rightarrow D$ and a causality relation $\preceq_{k}$ for which the following properties hold:

- the causality relation $\preceq_{k}$ is $\frac{1}{k}$-close to the Minkowski causality relation $\leq$;

- the bijection $f_{k} \frac{1}{k}$-preserves the relation $\preceq_{k}$; and

- the bijection $f_{k}$ is not $\varepsilon$-conformal, i.e., $d\left(f_{k}, \mathcal{C}\right) \geq \varepsilon$.

$2^{\circ}$. Similarly to the proof of Proposition 1, from this sequence, we can extract a subsequence $f_{k}$ which converges to some $m$-continuous bijection $f$. We will denote this subsequence by the same notations $f_{k}$. Then:

- $f_{k} \rightarrow f$

- $f_{k}^{-1} \rightarrow f^{-1}$

- each mapping $f_{k} \frac{1}{k}$-preserves the causality relation $\preceq_{k}$; and

- each mapping $f_{k}$ is not $\varepsilon$-conformal, i.e., $d\left(f_{k}, \mathcal{C}\right) \geq \varepsilon$.

$3^{\circ}$. Let us show that the limit transformation $f$ preserves the Minkowski causality relation $\leq$, i.e., that for all $a$ and $b$ for which $a \leq b$, we have $f(a) \leq f(b)$ and $f^{-1}(a) \leq f^{-1}(b)$.

$4^{\circ}$. Let us first prove the first implication. Let us fix $a$ and $b$ for which $a \leq b$.

$4.1^{\circ}$. Since the causality relation $\preceq_{k}$ is $\frac{1}{k}$-close to the Minkowski relation, $a \leq b$ implies that $a \frac{1}{k}$-approximately $\preceq_{k}$-precedes $b$, i.e., that there exist events $a_{k}$ and $b_{k}$ such that $\left\|a-a_{k}\right\| \leq 1 / k,\left\|b-b_{k}\right\| \leq 1 / k$, and $a \preceq_{k} b_{k}$.

$4.2^{\circ}$. Due to the fact that $f_{k} \frac{1}{k}$-preserves the causality relation $\preceq_{k}$, from $a_{k} \preceq_{k}$ $b_{k}$, we conclude that $f_{k}\left(a_{k}\right) \frac{1}{k}$-approximately $\preceq_{k}$-precedes $f_{k}\left(b_{k}\right)$, i.e., that there exist events $A_{k}$ and $B_{k}$ such that $\left\|A_{k}-f_{k}\left(a_{k}\right)\right\| \leq 1 / k,\left\|B_{k}-f_{k}\left(b_{k}\right)\right\| \leq 1 / k$, and $A_{k} \preceq_{k} B_{k}$. 
$4.3^{\circ}$. Since the causality relation $\preceq_{k}$ is $\frac{1}{k}$-close to the Minkowski relation, $A_{k} \preceq_{k}$ $B_{k}$ implies that $A_{k} \frac{1}{k}$-approximately $\leq$-precedes $b$, i.e., that there exist events $A_{k}^{\prime}$ and $B_{k}^{\prime}$ such that $\left\|A_{k}-A_{k}^{\prime}\right\| \leq 1 / k,\left\|B_{k}-B_{k}^{\prime}\right\| \leq 1 / k$, and $A_{k}^{\prime} \preceq_{k} B_{k}^{\prime}$.

4.4 ${ }^{\circ}$. From $\left\|a-a_{k}\right\| \leq 1 / k$ and $\left\|b-b_{k}\right\| \leq 1 / k$, we conclude that $a_{k} \rightarrow a$ and $b_{k} \rightarrow b$. Since the sequence $f_{k} \rightarrow f$ is equicontinuous, we conclude that $f_{k}\left(a_{k}\right) \rightarrow f(a)$ and $f_{k}\left(b_{k}\right) \rightarrow f(b)$.

$4.5^{\circ}$. Since $\left\|A_{k}-f_{k}\left(a_{k}\right)\right\| \leq 1 / k$ and $f_{k}\left(a_{k}\right) \rightarrow f(a)$, we conclude that the sequence $A_{k}$ tends the same limit $f(a)$. Since $\left\|A_{k}-A_{k}^{\prime}\right\| \leq 1 / k$, the sequence $A_{k}^{\prime}$ tends to the same limit as well: $A_{k}^{\prime} \rightarrow f(a)$.

Similarly, we have $B_{k}^{\prime} \rightarrow f(b)$. Since $A_{k}^{\prime} \leq B_{k}^{\prime}$ and the Minkowski causality relation is closed, $A_{k}^{\prime} \leq B_{k}^{\prime}$ implies that $\lim A_{k}^{\prime} \leq \lim B_{k}^{\prime}$, i.e., that $f(a) \leq f(b)$.

$5^{\circ}$. Similarly, we prove that $a \leq b$ implies $f^{-1}(a) \leq f^{-1}(b)$.

Thus, $f$ preserves the Minkowski causality and hence, according to Alexandrov's theorem, $f$ is a conformal mapping, i.e., $f \in \mathcal{C}$. This contradicts to our conclusion that $d(f, \mathcal{C}) \geq \varepsilon>0$. The proposition is proven.

Similar problem: transformations preserving a fixed distance. If instead of a space-time, we only consider a proper space $S$, then a natural question is: How can we measure a distance $d(a, b)$ between different points $a$ and $b$ in space - e.g., in the standard physical space $R^{3}$ ?

Ideally, we should have measuring instruments which can measure arbitrarily large and arbitrarily small distances. However, in reality, the sizes of the rulers are limited both from above (we cannot have too long rulers) and from below (we cannot have too short ones). Thus, the distances which can be directly measured by real rulers are also bounded from above and from below. A natural question is: if we only know such distances, can we uniquely determine the remaining ones?

In precise terms, let us assume that we have two number $\underline{d} \leq \bar{d}$ and we have a bijection mapping $f: S \rightarrow S$ for which, for all $a, b \in S$, if $\underline{d} \leq d(a, b) \leq \bar{d}$, then $d(f(a), f(b))=d(a, b)$. Will it then follows that $f$ is an isometry - and hence, for $S=R^{3}$, that $f$ is a linear metric-preserving transformation?

This is indeed true even for the case when $\underline{d}=\bar{d}$; see, e.g., $[6,9,10,17$, $28,34,36]$. For this case, the theorem says that every transformation which preserves a fixed distance $d$ is an isometry.

Definition 10. We say that a bijection $f: D \rightarrow D$ preserves distance 1 if it has the following two properties:

- for every two points $a, b \in D$, if $d(a, b)=1$, then $d(f(a), f(b))=1$, and

- for every two points $a, b \in D$, if $d(a, b)=1$, then $d\left(f^{-1}(a), f^{-1}(b)\right)=1$. 
Theorem. [6] Every bijection $f: R^{3} \rightarrow R^{3}$ which preserves distance 1 is a linear transformation.

A localized version of this result has been, in effect, proven in [21]:

Definition 11. By $B_{r} \stackrel{\text { def }}{=}\left\{x \in R^{3}: d(x, 0) \leq r\right\}$, we will denote a ball of radius $r$ with a center at 0 .

Proposition 3. [21] There exists a constant $C_{0}>1$ such that for every radius $r$, and for $R=C_{0} \cdot(r+1)$, every bijection $f: B_{R} \rightarrow B_{R}$ which preserves distance 1 is linear on $B_{r}$.

In principle, this result means that by measuring only distances from a limited range, we can uniquely reconstruct all the distances. However, from the practical viewpoint, this is not completely true. As before, measurements are never $100 \%$ accurate, so we can only get approximate values of the distance. So, the question is: if we want to know the distances with a given accuracy $\varepsilon>0$, is it possible to select a measurement accuracy $\delta>0$ in such a way that measurements with accuracy $\delta$ would enable us to reconstruct all the distances with the desired accuracy $\varepsilon$ ?

A construction similar to the one from causality preserving transformations shows that the result is "yes":

Definition 12. Let $\delta>0$. We say that a bijection $f: D \rightarrow D \delta$-preserves distance 1 if it has the following two properties:

- for every two points $a, b \in D$, if $d(a, b)=1$, then

$$
1-\delta \leq d(f(a), f(b)) \leq 1+\delta,
$$

and

- for every two points $a, b \in D$, if $d(a, b)=1$, then

$$
1-\delta \leq d\left(f^{-1}(a), f^{-1}(b)\right) \leq 1+\delta .
$$

Definition 13. Let $\varepsilon>0$ be a real number. We say that a function $f: D \rightarrow D$ is $\varepsilon$-linear if there exists a linear mapping $c: D \rightarrow D$ such that for every $a \in D$, we have $d(f(a), c(a)) \leq \varepsilon$.

Proposition 4. Let $C_{0}>1$ be a constant from Proposition 3, let $r>0$ be a given real number, let $R=C_{0} \cdot(r+1)$, and let $m$ be a modulus of continuity. Then, for every $\varepsilon>0$, there exists a $\delta>0$ such that if an $m$-continuous bijection $f: B_{R} \rightarrow B_{R} \delta$-preserves distance 1 , then $f$ is $\varepsilon$-linear on $B_{r}$.

The proof of this result is similar to the proof of Proposition 1. 
Comment. This proposition answers the question of whether it is potentially possible to reconstruct large or small distances by measuring medium ones. The next natural question is: How many direct measurements do we need for this reconstruction?

If we simply follow standard proofs from $[6,9,10,17,28,34,36]$, we get an unrealistic exponential number of measurements; a much faster (and thus realistic) measurement-and-reconstruction procedure is described in [21].

Acknowledgments. This work was supported in part by NSF grants EAR0225670 and EIA-0080940, by Texas Department of Transportation grant No. 0-5453, by the Japan Advanced Institute of Science and Technology (JAIST) International Joint Research Grant 2006-08, and by the Max Planck Institut für Mathematik.

The authors are thankful to U. Kohlenbach and G. Mints for valuable discussions.

\section{References}

[1] A. D. Alexandrov, "On Lorentz transformations", Uspekhi Math. Nauk, 1950, Vol. 5, No. 3 (37), p. 187 (in Russian).

[2] A. D. Alexandrov, "A contribution to chronogeometry", Canadian Journal of Mathematics, 1967, Vol. 19, pp. 1119-1128.

[3] A. D. Alexandrov, "Mappings of spaces with families of cones and spacetime transformations", Annali di Matematica Pura ed Aplicata, 1967, Vol. 103, pp. 229-257.

[4] A. D. Alexandrov, "On the axioms of relativity theory", Vestnik Leningrad University, Ser. Math., 1976, No. 19, pp. 5-28.

[5] A. D. Alexandrov and V. V. Ovchinnikova, "Remarks on the foundations of special relativity", Leningrad University Vestnik, 1953, No. 11, pp. 94-110 (in Russian).

[6] F. S. Beckman and D. A. Quarles Jr., "On isometries of Euclidean space", Proc. Amer. Math. Soc., 1953, Vol. 4, pp. 810-815.

[7] W. Benz, "A characterization of plane Lorentz transformations", Journal of Geometry, 1977, Vol. 10, pp. 45-56.

[8] W. Benz, Geometrische Transformationen, BI Wissenscahftsverlag, Mannheim, 1992.

[9] R. L. Bishop, "Characterizing motions by unit distance invariance", Mathematics Magazine, 1973, Vol. 46, pp. 148-151.

[10] D. Greenwell and P. D. Johnson Jr., "Functions that preserve unit distance", Mathematics Magazine, 1976, Vol. 49, No. 2, pp. 74-79. 
[11] A. K. Guts, "On mappings of families of sets", Soviet Math, Dokl., 1973, Vol. 14 , pp. 506-508.

[12] A. K. Guts, "On mappings preserving cones in a Lobachevski space", Math. Notes, 1973, Vol. 13, pp. 411-415.

[13] A. K. Guts, "Mappings of an ordered Lobachevsky space", Soviet Math. Dok., 1974, Vol. 15, pp. 416-419.

[14] A. K. Guts, "Invariant orders on three-dimensional Lie groups", Siberian Math. Journal, 1976, Vol. 17, pp. 731-736.

[15] A. K. Guts, "The chronogeometry of Gödel and de Sitter manifolds", Siberian Math. Journal, 1980, pp. 509-513.

[16] A. K. Guts, "Axiomatic relativity theory", Russian Math. Surveys, 1982, Vol. 37, No. 2, pp. 41-89.

[17] P. D. Johnson Jr., "The choice number of the plane", Geombinatorics, 1994, Vol. 3, No. 4, pp. 122-128.

[18] U. Kohlenbach, "Effective bounds from proofs in abstract functional analysis", In: B. Cooper, B. Loewe, and A. Sorbi (eds.), New Computational Paradigms: Changing Conceptions of What is Computable, Springer Verlag, Berlin-Heidelberg-New York, 2007.

[19] U. Kohlenbach, Applied Proof Theory: Proof Interpretations and their Use in Mathematics, Springer Verlag, Berlin-Heidelberg, 2008.

[20] O. Kosheleva and V. Kreinovich, "Unit-distance preserving theorem is logically non-trivial", Geombinatorics, 1995, Vol. 4, No. 4, pp. 119-128.

[21] O. Kosheleva and V. Kreinovich. "How to measure arbitrary distances using a given standard length (i.e., a stick with two marks on it): it is necessary, it is theoretically possible, it is feasible", Geombinatorics, 1996, Vol. 5, No. 4, pp. 142-155.

[22] O. M. Kosheleva, V. Kreinovich, and P. G. Vroegindewey, "An extension of a theorem of A. D. Alexandrov to a class of partially ordered fields", Proceedings of the Royal Academy of Science of Netherlands, 1979, Vol. 82(3), Series A, September 21, pp. 363-376.

[23] O. M. Kosheleva, V. Kreinovich, and P. G. Vroegindewey. Note on a physical application of the main theorem of chronogeometry, Technological University, Eindhoven, Netherlands, 1979, 7 pp.

[24] V. Kreinovich. "Approximately measured causality implies the Lorentz group: Alexandrov-Zeeman result made more realistic". International Journal of Theoretical Physics, 1994, Vol. 33, No. 8, pp. 1733-1747. 
[25] V. Kreinovich and O. Kosheleva, "An application of logic to combinatorial geometry: how many tetrahedra are equidecomposable with a cube?". Mathematical Logic Quarterly, 1994, Vol. 40, No. 1, pp. 31-34.

[26] A. V. Kuzminykh, "Characterization of Lorentz transformations", Soviet Math. Dokl., 1975, Vol. 16, No. 6, pp. 1626-1628.

[27] A. V. Kuzminykh, "Minimal condition determining the Lorentz transformations", Siberian Mathematical Journal, 1976, Vol. 17. No. 6, pp. 968-972.

[28] A. V. Kuz'minykh, "About a problem concerning functions which preserve unit distance", Geombinatorics, 1994, Vol. 3, No. 4, pp. 137-139.

[29] J. A. Lester, "On null cone preserving mappings", Proceedings of Cambridge Mathematical Society, 1977, Vol. 81, pp. 455-462.

[30] J. A. Lester, "Cone preserving mappings for quadratic cones over arbitrary fields", Canadian Journal of Mathematics, 1977, Vol. 29, pp. 1247-1253.

[31] J. A. Lester, "A physical characterization of conformal transformations of Minkowski spacetime", Annals of Discrete Mathematics, 1983, Vol. 18, pp. $567-574$.

[32] J. A. Lester, "Distance preserving transformations", In: F. Buekenhout (ed.), Handbook of Incidence Geometry, Elsevier, Amsterdam, 1995.

[33] G. L. Naber, The geometry of Minkowski space-time, Springer-Verlag, N.Y., 1992.

[34] C. G. Townsend, "Congruence-preserving mappings", Mathematics Magazine, 1970, Vol. 43, pp. 37-38.

[35] E. C. Zeeman, "Causality implies the Lorentz group", Journal of Mathematical Physics, 1964, Vol. 5, pp. 490-493.

[36] P. Zvengrowski, "Appendix to Chapter II", In: P. S. Modenov and A. S. Parkhomenko, Geometric Transformations, Vol. 1, Academic Press, N.Y., 1965, pp. 152-156. 